\title{
Exploration on Application of Electronic Circuit Simulation Technology in Electronic Application Technology
}

\author{
Mingming Fang \\ Weihai Vocational College, Weihai Shandong, 264210, China
}

\begin{abstract}
Key words: Electronic circuit simulation technology, Electronic application development, Application.
\end{abstract}

\begin{abstract}
The development of economy and society drives continuous progress of electronic technique, and electronic circuit simulation technology level gradually improves. In practical operation process, electronic circuit simulation technology is required to guarantee high efficiency of electronic development. In this way, electronic circuit simulation technology can be better applied in electronic product development. This paper analyzes the application of electronic circuit simulation technology in electronic product development, highlights the applications of electronic circuit simulation technology in virtual test circuit feature detection, circuit function verification, electronic product research expansion, promotion of integrated circuit development and realization of circuit design innovation and optimization. Finally, this paper expects the development of electronic circuit simulation technology and hopes to provide help for researchers.
\end{abstract}

\section{Introduction}

Social progress makes the degree of modernization higher and higher, while the development of economic technology makes new electronic products emerge in endlessly like mushrooms after rain. These products not just provide life convenience for people, but also improve people's living quality and enrich people's life. High-speed development of electronic products makes elite management level of electronic product enterprises improve. However, upgrading speed of electronic products is very fast. New technology makes upgrading time of electronic products is halved greatly. Thus, technical personnel of electronic products are keeping innovation and trying to change the development mode of electronic products so as to improve the quality of new electronic products. In the development process, development scientificity and safety of electronic products can be guaranteed only when electronic circuit simulation technology is fully applied. Electronic circuit simulation technology can achieve optimization and processing of electronic product design scheme and plays a great role in trail operation of electronic products [1]. If electronic circuit simulation technology cannot be rationally applied, operational defect and other problems may occur before electronic products come out. If unqualified electronic products are launched in the market, they will not just bring troubles to users, but also affect the reputation of electronic product enterprises. Thus, electronic circuit simulation technology is crucial for electronic application development.

\section{Application analysis}

(1) Application of electronic circuit simulation technology in virtual test circuit feature. Through electronic circuit simulation technology, diversified work environment can be better simulated in computer network operation process, and limit parameter environment for equipment operation can be provided so that extreme work state can be achieved more easily. For example, in actual operation process, the work environment of large current parameter can be simulated through rational use of 
electronic circuit simulation technology. In addition, electronic circuit test under high voltage and high temperature can be achieved. In actual circuit work state, it is very hard to reach repeated online of simulation technology, and electronic circuit cannot be guarantee to be in extreme work state anytime. Hence, electronic circuit simulation technology plays a crucial role for practical production and design of electronic circuit [2]. Electronic circuit simulation technology as the main basis of electronic product application development can not only achieve scientific design of electronic products, but also guarantee economical efficiency and safety of electronic circuit application.

(2) Application of electronic circuit simulation technology in verifying circuit functions. When electronic workers design electronic products as required by customers, it is necessary to confirm the design scheme and apply electronic circuit simulation technology to verify circuit functions according to actual operation environment of design scheme. When electronic circuit simulation technology is applied to verify the circuit, it can test whether the designed electronic circuit meets actual standard demand. Besides, in electronic product development and design process, electronic simulation technology can be used to identify whether the designed electronic product system has actual application effect and achieve functional error test of the designed circuit. Therefore, electronic circuit simulation technology runs through electronic application development process and has important significance for electronic application development. Reasonable application of electronic circuit simulation technology can reduce designers' design errors in electronic product development process. Before mass production of electronic products, scientific simulation is very necessary. It can detect the faults of electronic products, guarantee functional reliability of electronic products and finally reduce much follow-up unnecessary maintenance work [3]. Therefore, electronic circuit simulation technology can not only achieve high-quality production of electronic products, but also furthest reduce research and development time of electronic products and guarantee high efficiency of electronic product design.

(3) Electronic circuit simulation technology can help expand the research and development mode of electronic products. To ensure the quality of electronic products, practical research personnel pay more attention to the service time of electronic products, so a full set of electronic product development process is very complicated. A set of design scheme often needs repeated deliberations and repeated fabrications. If modification and adjustment of electronic product design scheme are not done fully in product design process, the designed electronic products cannot practically reach the design purpose, and even a large batch of unqualified products will occur. Thus, electronic circuit simulation technology is quite important for electronic design scheme modification and electronic circuit debugging. Compared with traditional circuit modification and circuit debugging, electronic circuit simulation technology has very prominent advantages. It can not only guarantee the accuracy of electronic design scheme modification and electronic circuit debugging, but also enhance work efficiency of electronic design scheme modification and electronic circuit debugging. Thus, to protect reliability of electronic products, they cannot be limited to traditional circuit modification and debugging. It is required to advance with the times, bring innovation to electronic product development technology according to practical circuit requirement and apply a new mode to ensure development scientificity of electronic products.

(4) Electronic circuit simulation technology can effectively promote the development of integrated circuit. In electronic product development, it is necessary to rationally apply electronic circuit simulation technology in order to facilitate healthy development of integrated circuit. Electronic products have very high requirements for integrated circuit in the development process. As the functions of electronic products improve, the density of integrated circuit also increases. Thus, electronic circuit designers need to transform development thought and apply chip-level system to design and develop electronic products. The essence of chip-level system application is to integrate all necessary functions in the chip [4]. This can not just guarantee application reliability of electronic products, but also effectively improve work efficiency of electronic products and meet actual operation needs. In order to do the above well, it is also necessary to rationally use electronic circuit simulation technology and apply it in electronic product development. 
(5) Electronic circuit simulation technology can achieve innovation and optimization of the circuit. Since the precision degree of electronic products is very high, they are quite sensitive to temperature. If the temperature of electronic products changes significantly, dysfunction phenomenon will occur to electronic products. Moreover, temperature change will also lead to serious restriction of work stability of electronic products. Hence, if electronic circuit simulation technology is applied, poor state of electronic product circuit can be changed in the initial stage of electronic product design. Since electronic products are influenced by the temperature, electronic circuit simulation technology can be used to simulate work state of electronic products under different temperature. Meanwhile, the features of electronic products can be analyzed according to the work state under different temperature. Designers of electronic products can analyze results according to electronic circuit simulation technology and improve electronic products according to the analysis results, which can furthest avoid temperature influence. If electronic products of the same type have different electronic parameters, serious problems will be caused to the production of electronic products. If electronic circuit simulation technology is rationally applied in the detection of electronic products, these problems can be handled reliably. ${ }^{[5]}$ This is because electronic circuit simulation technology can achieve systematical analysis of electronic products and gain corresponding analysis data. The designers can carry out optimization design of the circuit according to data results and ensure design scheme feasibility. For instance, tracking times can be set in the design process. Meanwhile, the parameters which fail to keep consistent in the electronic products can be analyzed. Finally, the simulation technology result can be used to modify the design scheme of electronic products.

\section{Development of electronic circuit simulation technology}

Continuous development of computer technology promotes the progress of electronic circuit simulation technology. Electronic circuit simulation technology has become a key field of computer development. So, we need to keep improvement of electronic circuit simulation technology and make electronic application development technology gain sustainable development. However, current electronic circuit simulation technology is still in the initial development stage and rests on simulation circuit hardware system query. CPU simulation cannot be achieved according to digital procedure corresponding to the hardware system. The researchers in the field of electronic science field are devoted to development and application of electronic circuit simulation technology. Driven by the development of China's science and technology, the algorithm of electronic circuit simulation technology will be increasingly accurate. Electronic circuit simulation technology which is applied in electronic circuit simulation system model will get further development, and electronic circuit simulation technology of CPU digital system procedure can be achieved.

CPU simulation planning of electronic circuit simulation technology is actually real-time simulation of embedded electronic system application program in electronic system simulation process. At the same time, real-time monitoring and test are implemented according to embedded electronic system application program and the work state in the circuit system. This is also the major direction to realize long-term development of electronic system. Secondly, we can achieve design limitations of hardware system and software system in future development of computer application system in accordance with CPU program of electronic circuit simulation technology and language description of corresponding hardware equipment. The development personnel of electronic application preprogram need to apply hardware description language to achieve design optimization of hardware platform and make electronic circuit simulation technology can be fully applied in follow-up design of application system. It is necessary to apply electronic circuit simulation technology to develop hardware equipment and relevant programs, do well design system accuracy calculation and reliable simulation, and finally discus with manufacturers with professional level about the entire scheme of electronic application system which passes the test. The design sample of standard electronic products which meet user demand can be gained according to production technology of electronic products. 
The development and application of electronic application technology is a process of transforming scientific, reliable and safe electronic design idea to electronic products and also the process of socializing and popularizing electronic products. The function of electronic circuit simulation technology is reflected in the application and development of electronic products. The sufficient application of hardware description language makes collaborative production mode of electronic products more diversified. Finally, large-scale development of integrated circuit of electronic products and wide application of electronic products are realized. Rational use of electronic circuit simulation technology can bring huge effects on the whole production process of electronic products. So, we need to enrich design means of electronic products in application development and use the products with high efficiency and high convenience to improve reliability of electronic products in order to better develop and utilize electronic application technology. For sound development of electronic product application development technology, it is necessary to focus on electronic application and development features, gradually improve electronic circuit simulation technology and make relevant test means of electronic circuit simulation technology more reliable and safer. Ultimately, the purposes of reducing development time of electronic products, improving development efficiency of electronic products and increasing development income of electronic products. In this way, electronic circuit simulation technology can be effectively applied in electronic application development technology.

\section{Conclusion}

In conclusion, in the whole process of achieving electronic application development, electronic circuit simulation technology plays a decision role. Hence, reasonable application of electronic circuit simulation technology in electronic application development has great significance. electronic circuit simulation technology as a widely-used and new electronic application development technology has broad development space. But, in practical electronic product application and production process, most people fail to really realize the importance of electronic circuit simulation technology, which restricts efficient development of electronic products. Hence, we need to let more and more electronic product developers fully know the benefits of electronic circuit simulation technology so as to drive the development of electronic product development field. This paper analyzes the specific application of electronic circuit simulation technology. In electronic product development, electronic circuit simulation technology can not just achieve detection of virtual test circuit features, but also verify circuit functions. Besides, electronic circuit simulation technology can help expand research and development mode of electronic products and enrich development approaches of electronic products. In terms of promoting integrated circuit development, electronic circuit simulation technology also plays certain role. Designers can use electronic circuit simulation technology to effectively achieve innovation and optimization of the circuit according to the operation condition of current circuit design scheme and then realize innovative development of electronic circuit simulation technology.

\section{References}

[1] Cao Jun, Study on Functions of Electronic Circuit Simulation Technology in Electronic Application Development, Electronic Test, 2016(12).

[2] Chen Zhe, Gao Chunwang, Zhang Junhe, Functions of Electronic Circuit Simulation Technology in Electronic Application Development, Heilongjiang Science and Technology Information, 2015(23).

[3] Fu Xiaofen, Analysis of Functions of Electronic Circuit Simulation Technology in Electronic Application Development, Discovering Value, 2013(14):72-72. 
[4] Du Jiang, Application of Electronic Circuit Simulation Technology in Electronic Application Development, Science Guide, 2013(8).

[5] Feng Weifeng, On Application of Electronic Circuit Simulation Technology in Electronic Application Development, Electronic Test, 2016(12). 